\title{
Higher-derivative non-Abelian gauge fields via the Faddeev-Jackiw formalism
}

\author{
R. Bufalo ${ }^{1,2, a}$, B. M. Pimentel ${ }^{2, b}$ \\ ${ }^{1}$ Department of Physics, University of Helsinki, P.O. Box 64, 00014 Helsinki, Finland \\ ${ }^{2}$ Instituto de Física Teórica (IFT), UNESP, São Paulo State University, Rua Dr. Bento Teobaldo Ferraz 271, Bloco II Barra Funda, São Paulo, \\ SP, CEP 01140-070, Brazil
}

Received: 12 June 2014 / Accepted: 21 July 2014 / Published online: 7 August 2014

(C) The Author(s) 2014. This article is published with open access at Springerlink.com

\begin{abstract}
In this paper we analyze two higher-derivative theories, the generalized electrodynamics and the AlekseevArbuzov-Baikov effective Lagrangian from the point of view of the Faddeev-Jackiw symplectic approach. It is shown that the full set of constraints is obtained directly from the zeromode eigenvectors, and that they are in accordance with wellknown results from Dirac's theory, a recurrent issue in the literature. The method shows to be rather economical in relation to the Dirac one, obviating thus unnecessary classification and calculations. Afterwards, to conclude we construct the transition amplitude of the non-Abelian theory following a constrained BRST method.
\end{abstract}

\section{Introduction}

A standard classical treatment of constrained theories was given originally by Dirac [1,2], it essentially analyzes the canonical structure of any theory, and it has been widely used in a great variety of quantum systems. However, it should be realized that Dirac's methodology is unnecessarily cumbersome and can be streamlined. Within this context, Faddeev and Jackiw [3] suggested a symplectic approach for constrained systems based in a first-order Lagrangian. This method has some very interesting properties of obviating the constraint classification, unnecessary calculations and the hypothesis of Dirac's conjecture as well. The FaddeevJackiw (FJ) symplectic formalism has been studied in a systematic way in different scenarios, shedding new light on the research of constrained dynamics.

The basic geometric structure of the Faddeev-Jackiw theory can be read directly from the elements of the inverse symplectic matrix, and they coincide with the correspondent

\footnotetext{
a e-mail: rodrigo.bufalo@helsinki.fi

b e-mail: pimentel@ift.unesp.br
}

Dirac brackets, providing thus a bridge to the commutators of the quantized theory. On the other hand, the results obtained from the Faddeev-Jackiw approach have been compared with the corresponding results of the Dirac method in different situations, for unconstrained and constrained systems, but it is still matter of study.

The method has as the key ingredient that these constraints produce deformations in the two-form symplectic matrix in such a way that, when all constraints are considered (by means of a Darboux transformation), the symplectic matrix is non-singular. As a result, one obtained the Dirac brackets. Nevertheless, it is important to emphasize that sometimes it happens that the (iteratively deformed ${ }^{1}$ ) two-form matrix is singular and no new constraint is obtained from the corresponding zero-mode. This is the case when one deals with gauge theories. At this point one should introduce convenient gauge (subsidiary) conditions, like a constraint; and the twoform matrix becomes, therefore, invertible. This extension was proposed and developed by Barcelos-Neto and Wotzasek [4,5] and by Montani and Wotzasek [6], and this was studied in several models [7-10]. It basically is in the spirit of Dirac's work, with proposal works by imposing the stability of the constraints under time evolution. So, constraints are not solved but embedded in an extended phase space. This is a more suitable approach when some relevant symmetries must be preserved.

A subtle issue subsequent to the Faddeev-Jackiw method is its equivalence to the Dirac method. Initially the equivalence was discussed in cases when the systems have no constraints $[11,12]$; but, in a constrained system, the situation was not completely clear, and some argumentation was provided earlier $[13,14]$ as regards the equivalence between the methods. However, recently was presented a proof [15] that

\footnotetext{
1 Actually the geometric role played by the constraints is to produce a 'deformation' in the original, singular, symplectic two-form matrix.
} 
the usual Faddeev-Jackiw method and Dirac method were not completely equivalent; namely, one showed that some constraints calculated in the Dirac formalism do not appear in the calculation in the Faddeev-Jackiw formalism. Then these would result in a contradiction between the usual FaddeevJackiw quantization and the Dirac quantization $[16,17]$.

Higher-derivative Lagrangian functions [18-20] are a fairly interesting branch of the ongoing effective theories; they were initially proposed as an attempt to enhance and render a better ultraviolet behavior of physically relevant models. It is well known that higher-derivative theories have, as a field theory, better renormalization properties than the conventional ones. These properties have shown to be quite appealing in the attempts to have a quantized and renormalizable theory of gravity [21-24]. The undesired features of the higher-derivative theory is that they possess a Hamiltonian that is not bounded from below and that the process of adding such terms jeopardizes the unitarity of the theory $[25,26]$. Besides all these motivations we emphasize that, from a theoretical point of view, higher-derivative theories have many interesting features that justify their study in itself.

As it has been pointed out in several works [27-31] over the years, it has been clear for a long time that Maxwell's theory is not the only one to describe the electromagnetic field. One of the most successful generalizations is the generalized electrodynamics [27-29]. Actually, Podolsky's theory is the only one that is a linear, Lorentz, and $U(1)$ invariant generalization of Maxwell's theory [31]. Another interesting feature inherent to Podolsky's theory is the existence of a generalized gauge condition also, namely, the generalized Lorenz condition: $\Omega[A]=\left(1+M^{-2} \square\right) \partial_{\mu} A^{\mu}$; considered an important issue, it is only through the choice of the correct gauge condition that we can completely fix the gauge degrees of freedom of a given gauge theory [30]. The relative success of these achievements motivated some authors to propose finite extensions of Quantum Chromodynamics (QCD) [3234] and also to advocate that higher order terms would be able to explain quark confinement. Our main goal here would be exactly to study both higher-derivative theories, Podolsky's electrodynamics and a non-Abelian $[35,36]$ extension of the model, also known as the Alekseev-Arbuzov-Baikov effective Lagrangian [37] in the framework of the Faddeev-Jackiw symplectic approach. So far we have no knowledge of application of the Faddeev-Jackiw method to higher-derivative theories. Moreover, this study may also shed some new light on the issue of whether the accordance between the Dirac and Faddeev-Jackiw methods holds.

In this paper, we discuss the canonical structure of the Podolsky electrodynamics and the $S U(N)$ AlekseevArbuzov-Baikov effective Lagrangian in the light of the Faddeev-Jackiw approach. In Sect. 2 we start by making a brief review of both the FJ and (constrained) symplectic formalisms. As the generalized electrodynamics of Podolsky has already been subject of analysis from the Dirac point of view [30], we shall study the theory via the FJ method in order to present an exercise of the methodology and also to check its consistency. Next, in Sect. 3, we discuss and introduce the Alekseev-Arbuzov-Baikov effective Lagrangian by discussing the generalized electrodynamics by making use of an enlargement of the gauge group to non-Abelian ones. Having defined the Lagrangian density we proceed by presenting the methodology, and obtaining the full set of constraints of the theory. Although an attempt of a path-integral formulation based on the FJ method has been proposed [38,39], there is no conclusive nor a clear argument to show the consistence of the method. Therefore, by means of complementarity of the previous discussion, we shall conclude the section by constructing the transition amplitude for the non-Abelian theory via the Batalin-Fradkin-Vilkovisky (BFV) method [40-42], obtaining an important result for subsequent analysis in the quantum level. In Sect. 4 we summarize the results, and present our final remarks and prospects.

\section{Generalized electrodynamics via Faddeev-Jackiw formalism}

\subsection{Faddeev-Jackiw symplectic method}

Let us start with a first-order in time derivative Lagrangian, which may arise from a conventional second-order one after introducing auxiliary fields. First, one can construct the symplectic Lagrangian ${ }^{2}$

$\mathscr{L}=a_{i}(\xi) \dot{\xi}^{i}-\mathscr{V}(\xi)$

with the arbitrary one-form components $a_{i}$, with $i=$ $1, \ldots, N$. The first-order system is characterized by a closed two-form. If the two-form is non-degenerate, it defines a symplectic structure on the phase space, described by the coordinates $\xi_{i}$. On the other hand, if the two-form is singular, with constant rank, it is called a pre-symplectic two-form. Thus, in terms of components, the (pre)symplectic form is defined by

$f_{i j}=\frac{\partial}{\partial \xi^{i}} a_{j}(\xi)-\frac{\partial}{\partial \xi^{j}} a_{i}(\xi)$.

The Euler-Lagrange equations are given by

$f_{i j} \dot{\xi}^{j}=\frac{\partial}{\partial \xi^{i}} \mathscr{V}(\xi)$

\footnotetext{
${ }^{2}$ In this section we discuss a system with finite degrees of freedom. However, an extension to the infinite degrees of freedom case can be attained in a straightforward way.
} 
Now, when the two-form $f_{i j}$ is non-singular, it has an inverse $f^{i j}$, then

$\dot{\xi}^{i}=f^{i j} \frac{\partial}{\partial \xi^{j}} \mathscr{V}(\xi)$,

and the basic brackets are defined as $\left\{\xi^{i}, \xi^{j}\right\}=f^{i j}$. However, in the case that the Lagrangian (1) describes a constrained system, the symplectic matrix is singular, and the constraints hidden in the system need to be determined. Let us suppose that the rank of $f_{i j}$ is $2 n$, so there is $N-2 n=M$ zero-mode vectors $\mathbf{v}^{\alpha}, \alpha=1, \ldots, M$. The system is then constrained by $M$ equations in which no time-derivatives appear. Then there will be constraints that reduce the number of degrees of freedom. Thus, multiplying (3) by the (left) zero-modes $\mathbf{v}^{\alpha}$ of $f_{i j}$ we get the (symplectic) constraints in the form of algebraic relations

$\Omega^{\alpha} \equiv \mathbf{v}_{i}^{\alpha} \frac{\partial}{\partial \xi^{i}} \mathscr{V}(\xi)=0$.

Then one can give the first-iterated Lagrangian by introducing corresponding Lagrange multipliers of the obtained constraints

$\mathscr{L}=a_{i}^{(1)}(\xi) \dot{\xi}^{i}+\Omega^{\alpha} \lambda_{\alpha}-\mathscr{V}^{(1)}(\xi)$.

Hence, one may regard the introduced Lagrange multipliers $\lambda$ as symplectic variables and can extend the symplectic variable set. This procedure reduces the number of $\xi$ 's. Then the whole procedure can be repeated again until all constraints are eliminated and we are left with a completely reduced, unconstrained, and canonical system. However, it should be remarked that in the case of gauge theories, the zero-mode does not give any new constraint (it still does not give the full rank matrix), and the symplectic matrix remains singular. Thus, we should consider that it is necessary to introduce gauge condition(s) to obviate the singularity. So, the work can be finished in expectation in terms of the original variables, and the basic brackets can be determined.

\subsection{Generalized electrodynamics}

The purpose of the present study is to examine the FJ methodology applied in the analysis of a higher-derivative theory. It is interesting to study first, as a simpler example, an Abelian theory. Therefore, in order to keep things simple, we choose the simplest but rather interesting Abelian electrodynamics of Podolsky, whose Lagrangian density is given by

$\mathscr{L}=-\frac{1}{4} F^{\mu \nu} F_{\mu \nu}+\frac{1}{2 M^{2}} \partial_{\mu} F^{\mu \nu} \partial^{\lambda} F_{\lambda \nu}$,

where $F_{\mu \nu}=\partial_{\mu} A_{\nu}-\partial_{\nu} A_{\mu}$ and the spacetime metric elements are $\eta_{\mu v}=(1,-1,-1,-1)$. It should be mentioned that we shall follow the Ostrogradski approach [18-20] to deal with the higher-derivative terms. Hence, there should be introduced another set of the canonical pair $\left(\Gamma^{\mu} \equiv\right.$ $\partial_{0} A^{\mu}, \phi_{\nu}$ ) in order to have a correct expanded phase space to thus proceed with the canonical analysis. With this thought in mind one finds the following Lagrangian [30]:

$$
\begin{aligned}
\mathscr{L}= & \frac{1}{2}\left(\vec{\Gamma}-\nabla A_{0}\right)^{2}+\frac{1}{2}(\nabla \times \vec{A})^{2} \\
& +\frac{1}{2 M^{2}}\left[\left(\nabla \cdot \vec{\Gamma}-\nabla^{2} A_{0}\right)^{2}\right. \\
& \left.-\left(\partial_{0} \vec{\Gamma}-\nabla \Gamma_{0}-\nabla \times(\nabla \times \vec{A})\right)^{2}\right] .
\end{aligned}
$$

To transform this Lagrangian from second to first order, we shall use an auxiliary field, which is chosen to be the canonical momentum due to an algebraic simplification that it provides. In that case, we should recall that we have an additional set of canonical pairs, in particular here, $(A, \pi)$ and $(\Gamma, \phi)$ :

$\phi^{\mu}=\frac{\partial \mathscr{L}}{\partial\left(\partial_{0} \partial_{0} A_{\mu}\right)}$,

and

$\pi^{\mu}=\frac{\partial \mathscr{L}}{\partial\left(\partial_{0} A_{\mu}\right)}-2 \partial_{k}\left(\frac{\partial \mathscr{L}}{\partial\left(\partial_{0} \partial_{k} A_{\mu}\right)}\right)-\partial_{0}\left(\frac{\partial \mathscr{L}}{\partial\left(\partial_{0} \partial_{0} A_{\mu}\right)}\right)$,

resulting in the following expressions:

$\pi^{\mu}=F^{\mu 0}-M^{-2}\left(\eta^{\mu k} \partial_{k} \partial_{\lambda} F^{0 \lambda}-\partial_{0} \partial_{\lambda} F^{\mu \lambda}\right)$,

and

$M^{2} \phi^{\mu}=\left(\eta^{\mu 0} \partial_{\lambda} F^{0 \lambda}-\partial_{\lambda} F^{\mu \lambda}\right)$,

where

$M^{2} \vec{\phi}=\partial_{0} \vec{\Gamma}-\nabla \Gamma^{0}-(\nabla \times(\nabla \times \vec{A}))$.

In order to obtain the quadratic kinetic terms we may make use of the equation of motion for $\phi$ (12) back into the Lagrangian. Therefore, in this case one can cast the Lagrangian density (8) in the form

$$
\mathscr{L}=-\phi_{k} \dot{\Gamma}_{k}+\pi_{\mu} \dot{A}^{\mu}-\mathscr{V}^{(0)}
$$

where the potential density is

$$
\begin{aligned}
\mathscr{V}^{(0)}= & \pi_{\mu} \Gamma^{\mu}-\frac{1}{2}\left(\vec{\Gamma}-\nabla A_{0}\right)^{2}-\frac{1}{2}(\nabla \times \vec{A})^{2} \\
& -\frac{1}{2 M^{2}}\left(\nabla \cdot \vec{\Gamma}-\nabla^{2} A_{0}\right)^{2} \\
& -\frac{M^{2}}{2} \vec{\phi}^{2}-\vec{\phi} \cdot\left(\nabla \Gamma_{0}+\nabla \times(\nabla \times \vec{A})\right) .
\end{aligned}
$$


The initial set of symplectic variables is seen to be $\xi_{\alpha}^{(0)}=$ $\left\{A_{k}, \pi_{k}, A_{0}, \pi_{0}, \Gamma_{k}, \phi_{k}, \Gamma_{0}\right\}$; this permits us to identify the non-null canonical one-form

$\Gamma a_{i}^{(0)}=-\phi_{i}, \quad{ }^{A} a_{i}^{(0)}=-\pi_{i}, \quad{ }^{A_{0}} a^{(0)}=\pi_{0}$.

The previous results lead to the corresponding two-form matrix

${ }^{(0)} f_{a b}(x, y)=\left[\begin{array}{cc}\mathbf{A}_{i j} & \mathbf{0}_{\mathbf{4} \times \mathbf{3}} \\ \mathbf{0}_{\mathbf{3} \times \mathbf{4}} & \mathbf{B}_{i j}\end{array}\right] \delta(x, y)$,

with

$\mathbf{A}_{i j}=\left[\begin{array}{cccc}0 & \delta_{i j} & 0 & 0 \\ -\delta_{i j} & 0 & 0 & 0 \\ 0 & 0 & 0 & -1 \\ 0 & 0 & 1 & 0\end{array}\right], \quad \mathbf{B}_{i j}=\left[\begin{array}{ccc}0 & \delta_{i j} & 0 \\ -\delta_{i j} & 0 & 0 \\ 0 & 0 & 0\end{array}\right]$

and it is not difficult to see that the matrix is singular. Moreover, it is easy to determine that the eigenvector with zero eigenvalue is

$v_{\alpha}=\left(\mathbf{0}, \mathbf{0}, 0,0, \mathbf{0}, \mathbf{0}, v^{7}\right)$

where $v^{7}$ is arbitrary and associated to $\Gamma_{0}$. Therefore, from the eigenvector $v_{\alpha}$ (18) we can evaluate the consistence condition as

$$
\int \mathrm{d} x \mathrm{~d} y v^{7} \frac{\delta}{\delta \Gamma_{0}(x)} \mathscr{V}^{(0)}(y)=\int \mathrm{d} x v^{7}\left(\pi_{0}+\nabla \cdot \vec{\phi}\right)=0
$$

since $v^{7}(x)$ is an arbitrary function, we obtain the constraint

$\Omega(x) \equiv \pi_{0}(x)+\nabla \cdot \vec{\phi}(x)=0$

Introducing this constraint back into the Lagrangian by means of a Lagrange multiplier $\lambda^{3}$ we have

$\mathscr{L}=-\phi_{k} \dot{\Gamma}_{k}+\pi_{\mu} \dot{A}^{\mu}+\dot{\lambda}\left(\pi_{0}+\nabla . \vec{\phi}\right)-\mathscr{V}^{(1)}$,

where the first-iterated potential density is $\mathscr{V}^{(1)}=\left.\mathscr{V}^{(0)}\right|_{\Omega=0}$ with

\footnotetext{
${ }^{3}$ It should be noted that when the constraint $\Omega$ is imposed the dependence in $\Gamma_{0}$ naturally disappears.
}

$$
\begin{aligned}
\mathscr{V}^{(1)}= & -\pi_{k} \Gamma_{k}-\frac{1}{2}\left(\vec{\Gamma}-\nabla A_{0}\right)^{2}-\frac{1}{2}(\nabla \times \vec{A})^{2} \\
& -\frac{1}{2 M^{2}}\left(\nabla \cdot \vec{\Gamma}-\nabla^{2} A_{0}\right)^{2} \\
& -\frac{M^{2}}{2} \vec{\phi}^{2}-\vec{\phi} \cdot(\nabla \times(\nabla \times \vec{A})) .
\end{aligned}
$$

From the above Lagrangian we have the following vectors:

$$
\begin{gathered}
\Gamma a_{i}^{(1)}=-\phi_{i}, \quad{ }^{A} a_{i}^{(1)}=-\pi_{i}, \quad{ }^{A_{0}} a^{(1)}=\pi_{0}, \\
{ }_{a} a^{(1)}=\pi_{0}+\nabla \cdot \vec{\phi},
\end{gathered}
$$

and these results lead to the corresponding two-form matrix,

$$
{ }^{(1)} f_{a b}(x, y)=\left[\begin{array}{cc}
\mathbf{A}_{i j} & \mathbf{D}_{j} \\
-\mathbf{D}_{i}^{\mathbf{T}} & \mathbf{C}_{i j}
\end{array}\right] \delta(x, y) \text {, }
$$

with $\mathbf{C}(x, y)$ and $\mathbf{D}(x, y)$ being the Abelian version of the non-Abelian expressions $\mathbf{C}_{a b}(x, y)$ and $\mathbf{D}_{a b}(x, y),(63)$. We obtain once again a singular matrix. From that, we can determine its eigenvector with zero eigenvalue,

$\bar{v}_{\alpha}=\left(\mathbf{0}, \mathbf{0}, \bar{v}^{3}, 0, \bar{v}_{i}^{5}, \mathbf{0}, \bar{v}^{7}\right)$

Therefore, following the outlined routine, from this eigenvector $\bar{v}_{\alpha}$ (25) we can evaluate the consistence condition

$$
\begin{gathered}
\int \mathrm{d} x\left[\bar{v}^{3} \frac{\delta}{\delta A_{0}(x)}+\bar{v}_{i}^{5} \frac{\delta}{\delta \Gamma^{i}(x)}\right] \int \mathrm{d} y^{\mathscr{V}^{(1)}}(y) \\
=\int \mathrm{d} x \bar{v}^{3}(\nabla \cdot \vec{\pi})(x)=0,
\end{gathered}
$$

where in the last equality we have made use of the relation $\bar{v}_{i}^{5}-\partial_{i} \bar{\nu}^{3}=0$. Once again, as $\bar{v}^{3}$ is an arbitrary function, we obtain a new constraint relation (Gauss' law)

$\bar{\Omega}(x) \equiv(\nabla \cdot \vec{\pi})(x)=0$.

Now, following the methodology, the second-iterated Lagrangian reads

$\mathscr{L}=-\phi_{k} \dot{\Gamma}_{k}+\pi_{\mu} \dot{A}^{\mu}+\dot{\lambda}\left(\pi_{0}+\nabla \cdot \vec{\phi}\right)+\dot{\eta}(\nabla . \vec{\pi})-\mathscr{V}^{(2)}$,

whereas the second-iterated potential density is

$\mathscr{V}^{(2)}=\left.\mathscr{V}^{(1)}\right|_{\bar{\Omega}=0}=\mathscr{V}^{(1)}$

From the above Lagrangian one finds the following vectors:

$$
\begin{array}{r}
\Gamma a_{i}^{(2)}=-\phi_{i}, \quad{ }^{A} a_{i}^{(2)}=-\pi_{i}, \quad{ }^{A_{0}} a^{(2)}=\pi_{0}, \\
\lambda a^{(2)}=\pi_{0}+\nabla \cdot \vec{\phi}, \quad \eta a^{(2)}=\nabla \cdot \vec{\pi},
\end{array}
$$


these results lead to the corresponding two-form matrix

${ }^{(2)} f_{a b}(x, y)=\left[\begin{array}{cc}\mathbf{A}_{i j} & \mathbf{E}_{j, x} \\ -\mathbf{E}_{i, y}^{T} & \mathbf{F}_{i j}\end{array}\right] \delta(x, y)$,

with

$\mathbf{E}_{i, x}=\left[\begin{array}{cccc}0 & 0 & 0 & 0 \\ 0 & 0 & 0 & -\partial_{i}^{x} \\ 0 & 0 & 0 & 0 \\ 0 & 0 & 1 & 0\end{array}\right], \quad \mathbf{F}_{i j}=\left[\begin{array}{cccc}0 & \delta_{i j} & 0 & 0 \\ -\delta_{i j} & 0 & -\partial_{i}^{x} & 0 \\ 0 & -\partial_{i}^{x} & 0 & 0 \\ 0 & 0 & 0 & 0\end{array}\right]$.

It follows that the second-iterated matrix ${ }^{(2)} f_{a b}(x, y)$ is also singular. Thus one obtains two zero-mode vectors,

$\tilde{v}_{\alpha}=\left(\tilde{v}_{i}^{1}, \mathbf{0}, 0,0, \mathbf{0}, \mathbf{0}, 0, \tilde{v}^{\mathbf{8}}\right)$

and

$\overline{\bar{v}}_{\alpha}=\left(\mathbf{0}, \mathbf{0}, \overline{\bar{v}}^{3}, 0, \overline{\bar{v}}_{i}^{5}, \mathbf{0}, \overline{\bar{v}}^{7}, \mathbf{0}\right)$.

However, the vector $\overline{\bar{v}}_{\alpha}$ generates the constraint $(\nabla \cdot \vec{\pi})=0$. Therefore, only the vector $\tilde{v}_{\alpha}$ is of interest. Consequently, the consistence condition results in

$$
\int \mathrm{d} x \mathrm{~d} y\left[\tilde{v}_{i}^{1}(x) \frac{\delta}{\delta A^{i}(x)}+\tilde{v}^{8}(x) \frac{\delta}{\delta \eta(x)}\right] \mathscr{V}^{(2)}(y)=0 .
$$

Thus, the zero mode does not generate any new constraints and, consequently, the symplectic matrix remains singular. This is an imprint characteristic of gauge theories, therefore, the gauge degrees of freedom have to be fixed. We choose to do the work here with the generalized Coulomb gauge: $A_{0}=0$ and $\left(1+M^{-2} \square\right)(\nabla \cdot \vec{A})=0 .{ }^{4}$ We then obtain a new Lagrangian density,

$$
\begin{aligned}
\mathscr{L}= & -\phi_{k} \dot{\Gamma}_{k}+\pi_{k} \dot{A}^{k}+\dot{\lambda}\left(\pi_{0}+\nabla \cdot \vec{\phi}\right)+\dot{\eta}(\nabla \cdot \vec{\pi}) \\
& +\dot{\chi}\left(1-M^{-2} \nabla^{2}\right) \nabla \cdot \vec{A}-\mathscr{V}^{(3)},
\end{aligned}
$$

where the third-iterated potential density is $\mathscr{V}^{(3)}=\left.\mathscr{V}^{(2)}\right|_{\bar{\Omega}=0}$ with

$$
\begin{aligned}
\mathscr{V}^{(3)}= & -\pi_{k} \Gamma_{k}-\frac{1}{2} \vec{\Gamma}^{2}+\frac{1}{2} \vec{A} \cdot\left(\nabla^{2} \vec{A}\right) \\
& -\frac{1}{2 M^{2}}(\nabla \cdot \vec{\Gamma})^{2}-\frac{M^{2}}{2} \vec{\phi}^{2}-\vec{\phi} \cdot\left(\nabla^{2} \vec{A}\right) .
\end{aligned}
$$

We have absorbed the $(\nabla \cdot \vec{A})$ terms into the new constraint. It is worth to emphasize that from the expression for the

\footnotetext{
${ }^{4}$ It is worth to mention that the complete generalized Coulomb gauge has in addition the condition: $\Gamma_{0}=0$, but as it has already disappeared in the Lagrangian, it is not necessary to impose it.
}

potential $\mathscr{V}^{(3)}$ one may read off which are the dynamical variables; for instance, here, it consists of the canonical set $\left\{A_{k}, \pi^{m}\right\}$ and $\left\{\Gamma_{k}, \phi^{m}\right\}$.

Nevertheless, from the above Lagrangian follow the vectors:

$$
\begin{gathered}
\Gamma a_{i}^{(3)}=-\phi_{i}, \quad{ }^{A} a_{i}^{(3)}=-\pi_{i}, \quad \lambda a^{(3)}=\pi_{0}+\nabla \cdot \vec{\phi}, \\
\eta^{(3)}=\nabla \cdot \vec{\pi}, \quad \chi a^{(3)}=\nabla_{P}^{2} \nabla \cdot \vec{A},
\end{gathered}
$$

where $\nabla_{P}^{2}=\left(1-M^{-2} \nabla^{2}\right)$; these lead to the corresponding third-iterated symplectic matrix, ${ }^{5}$

${ }^{(3)} f_{a b}(x, y)=\left[\begin{array}{cc}\mathbf{B}_{i j} & \mathbf{G}_{j, x} \\ -\mathbf{G}_{i, y}^{T} & \tilde{\mathbf{F}}_{i j, x}\end{array}\right] \delta(x, y)$

with

$\mathbf{G}_{i, x}=\left[\begin{array}{ccccc}0 & 0 & 0 & 0 & -\nabla_{P}^{2} \partial_{i}^{x} \\ 0 & 0 & 0 & -\partial_{i}^{x} & 0 \\ 0 & 0 & 1 & 0 & 0\end{array}\right]$

This ${ }^{(3)} f_{a b}(x, y)$ is clearly a non-singular matrix and the corresponding inverse is easily obtained by a simple but rather lengthy calculation. Moreover, we may relabel $\lambda=\phi_{0}$, $\eta=\Gamma_{0}$, and $\chi=A_{0}$. Therefore, the generalized brackets between the dynamical variables, the corresponding Dirac brackets in the generalized radiation gauge, are just the elements of the inverse of such a matrix, and they read

$$
\begin{aligned}
& \left\{A_{k}(x), \pi^{m}(y)\right\}^{\star}=\delta_{k}^{m} \delta(x, y)-\nabla_{P}^{2} \partial_{k} \partial^{m} G(x, y), \\
& \left\{\Gamma_{k}(x), \phi^{m}(y)\right\}^{\star}=\delta_{k}^{m} \delta(x, y),
\end{aligned}
$$

where we have introduced the Green function

$\nabla_{P}^{2} \nabla^{2} G(x, y)=\delta^{(3)}(x, y)$

These results are in accordance to those obtained previously through an analysis à la Dirac in [30]. Though we have obtained the correct brackets to the dynamic variables, we are left with the whole canonical variables (including the kinematical ones) without any trace of which variables are in fact dynamical and that, therefore, should be submitted to the quantization (a natural outcome of the Dirac theory). Nevertheless, the analysis of this particular theory showed that the outcomes of both theories match, although both present pros and cons, especially those involving unnecessary calculations and tedious algebraic work.

\footnotetext{
$\overline{5 \tilde{\mathbf{F}}_{i j, x} \text { is equal }}$ to the expression of $\mathbf{F}_{i j, x}$, (32), but with an additional fifth null line and column.
} 


\section{$3 S U(N)$ higher-derivative Yang-Mills-Utiyama theory}

In this section we will go a step further from the previous discussion and consider a non-Abelian extension of the Podolsky theory, also known as the Alekseev-Arbuzov-Baikov effective Lagrangian [37]. This theory was originally proposed as an attempt to eliminate the infrared divergences in $S U(N)$ theories [32-34]. In order to introduce some concepts, let us consider the $U(1)$ electrodynamics in four dimensions, see (7),

$\mathscr{L}=-\frac{1}{4} F_{\mu \nu} F^{\mu \nu}+\frac{1}{2 M^{2}} \partial_{\mu} F^{\mu \nu} \partial^{\lambda} F_{\lambda \nu}$.

Moreover, to make contact with the non-Abelian theory [35, 36], it is interesting to discuss an additional point. It is not difficult to see that it is still possible to add a second higherderivative term in (44), but in order to preserve the original dispersion relation we have

$k^{2}\left(k^{2}-M^{2}\right) A_{\mu}(k)=0$,

when the generalized condition $\left(k^{2}-M^{2}\right) k^{\mu} A_{\mu}(k)=0$ holds. Hence, the Lagrangian should be rewritten as

$\mathscr{L}=-\frac{1}{4} F_{\mu \nu} F^{\mu \nu}+\frac{1}{6 M^{2}} \partial_{\mu} F^{\mu \nu} \partial^{\lambda} F_{\lambda \nu}+\frac{1}{6 M^{2}} \partial_{\lambda} F^{\mu \nu} \partial^{\lambda} F_{\mu \nu}$,

since $\left(\partial_{\lambda} F^{\mu \nu}\right)^{2}=2\left(\partial_{\lambda} F^{\lambda v}\right)^{2}$. Therefore, the starting point of our analysis would be the Lagrangian density (46). Thus, to input an internal symmetry group, the original field must change as $A_{\mu} \rightarrow A_{\mu}^{a}$, where $a=1, \ldots,\left(N^{2}-1\right)$, denotes an index belonging to some internal symmetry group introduced into the original theory, in our case $S U(N)$. Assuming that $X=X^{a} \tau^{a}$, where $\tau^{a}$ are the generators of the corresponding Lie algebra, $\left[\tau^{a}, \tau^{b}\right]=i f^{a b c} \tau^{c}$, and that it transforms as an adjoint representation of the symmetry group, we rewrite the original Lagrangian density:

$$
\begin{aligned}
\mathscr{L}_{\mathrm{AAB}}= & -\frac{1}{4} W^{a}{ }_{\mu \nu} W^{a \mu \nu}+\frac{1}{6 M^{2}}\left(D_{\mu} W^{\mu \nu}\right)^{b}\left(D^{\sigma} W_{\sigma \nu}\right)^{b} \\
& +\frac{1}{6 M^{2}}\left(D_{\sigma} W^{\mu \nu}\right)^{a}\left(D^{\sigma} W_{\mu \nu}\right)^{a} \\
& -\frac{g}{18 M^{2}} f^{a b c} W_{\mu \nu}^{a} W^{b \nu \lambda} W_{\lambda \alpha}^{c} \eta^{\alpha \mu}
\end{aligned}
$$

where $W_{\mu \nu}^{a}$ is a non-Abelian stress tensor with the following form:

$W^{a}{ }_{\mu \nu}=F^{a}{ }_{\mu \nu}+g G^{a}{ }_{\mu \nu}$,

where $g$ is a group parameter and $G^{a}{ }_{\mu \nu}=f^{a b c} A_{\mu}^{b} A_{\nu}^{c}$, and we also see that the covariant derivative is given by $D_{\mu}^{a c} \equiv$ $\delta^{a c} \partial_{\mu}+g f^{a b c} A_{\mu}^{b}$, with $\left(\tau^{a}\right)^{b c}=-i f^{a b c}$.
Now, in order to carry out the second step of the method we should first rewrite the Lagrangian density (47) in its firstorder form. To accomplish that, we may use the canonical momenta due to an algebraic simplification in this choice. Therefore, from the definition (10) one may evaluate

$3 M^{2} \phi^{a \mu}=\left(D_{\sigma} W^{\sigma \mu}\right)^{a}-\eta^{0 \mu}\left(D_{\sigma} W^{\sigma 0}\right)^{a}+2\left(D^{0} W^{0 \mu}\right)^{a}$,

and it follows that

$3 M^{2} \phi^{a n}=\left(D_{m} W^{m n}\right)^{a}+3\left(D_{0} W^{0 n}\right)^{a}$.

With the above results in hands we may now rewrite the Lagrangian (47) in its first-order form in terms of the time derivatives of the field potential, ${ }^{6}$ and one then obtains the first-order Lagrangian

$\mathscr{L}=-\phi_{k}^{a} \dot{\Gamma}_{k}^{a}+\pi_{\mu}^{a} \dot{A}^{a \mu}-\mathscr{V}^{(0)}$,

whereas the potential density is

$$
\begin{aligned}
& \mathscr{V}^{(0)}=\pi_{\mu}^{a} \Gamma^{a \mu}-\frac{M^{2}}{2} \phi_{k}^{a} \phi_{k}^{a}+\phi_{k}^{a}\left(\frac{1}{3}\left(D^{m} W_{m k}\right)^{a}\right. \\
& \left.-\partial_{k} \Gamma_{0}^{a}+g f^{a b c}\left(\Gamma_{0}^{b} A_{k}^{c}+A_{0}^{b} \Gamma_{k}^{c}\right)+g f^{a b c} A_{0}^{b} W_{0 k}^{c}\right) \\
& -\frac{1}{6 M^{2}}\left(D_{0} W^{n m}\right)^{a}\left(D^{0} W_{n m}\right)^{a} \\
& +\frac{1}{4} W^{a}{ }_{k m} W^{a k m}-\frac{1}{2}\left(\Gamma_{k}^{a}-\partial_{k} A_{0}^{a}+g f^{a b c} A_{0}^{b} A_{k}^{c}\right)^{2} \\
& -\frac{1}{6 M^{2}}\left[\left(D_{r} W^{n m}\right)^{a}\left(D^{r} W_{n m}\right)^{a}+\left(D_{r} W^{r 0}\right)^{a}\left(D^{m} W_{m 0}\right)^{a}\right. \\
& \left.+\frac{2}{3}\left(D_{m} W^{m n}\right)^{a}\left(D^{r} W_{r n}\right)^{a}+2\left(D_{m} W^{0 r}\right)^{a}\left(D^{m} W_{0 r}\right)^{a}\right] \\
& +\frac{g}{18 M^{2}} f^{a b c}\left[3 W_{0 m}^{a} W_{m k}^{b} W_{k 0}^{c}-W_{k m}^{a} W_{m j}^{b} W_{j k}^{c}\right] .
\end{aligned}
$$

From the above expression (51) for the Lagrangian density one may read off the initial set of symplectic variables,

$\xi_{\alpha}^{(0)}=\left\{A_{k}^{a}, \pi_{k}^{a}, A_{0}^{a}, \pi_{0}^{a}, \Gamma_{k}^{a}, \phi_{k}^{a}, \Gamma_{0}^{a}\right\}$,

moreover, from (51) we can identify the non-null canonical one-form

$\Gamma a_{i}^{(0)}=-\phi_{i}^{a}, \quad{ }^{A} a_{i}^{(0)}=-\pi_{i}^{a}, \quad{ }^{A_{0}} a^{(0)}=\pi_{0}^{a}$.

From these results, we can compute the elements of the symplectic matrix, leading to the corresponding two-form matrix,

${ }^{6}$ We follow again the Ostrogradski formalism to deal with the higherderivative terms. 
${ }^{(0)} f_{a b}(x, y)=\left[\begin{array}{cc}\mathbf{A}_{i j}^{a b} & \mathbf{0}_{\mathbf{4} \times \mathbf{3}} \\ \mathbf{0}_{\mathbf{3} \times \mathbf{4}} & \mathbf{B}_{i j}^{a b}\end{array}\right] \delta(x, y)$,

with $\mathbf{A}_{a b}(x, y)$ and $\mathbf{B}_{a b}(x, y)$ having the same expression to the $\mathbf{A}(x, y)$ and $\mathbf{B}(x, y),(17)$, with an additional non-Abelian indexing $\delta^{a b}$. The matrix ${ }^{(0)} f_{a b}(x, y)$ is obviously singular. The eigenvector with zero eigenvalue is

$v_{\alpha}=\left(\mathbf{0}, \mathbf{0}, 0,0, \mathbf{0}, \mathbf{0}, v_{7}^{a}\right)$,

where $\nu_{7}$ is an arbitrary function, associated with $\Gamma_{0}^{a}$. Hence, from the eigenvector (56) we can calculate the consistence condition

$$
\int \mathrm{d} x v_{7}^{a}(x)\left[\pi_{0}^{a}+D_{k}^{a b} \phi_{k}^{b}\right](x)=0,
$$

and, since $v_{7}^{a}$ is an arbitrary function, we obtain the constraint

$\chi^{a}(x) \equiv \pi_{0}^{a}+D_{k}^{a b} \phi_{k}^{b}=0$.

Following the methodology, we should now introduce this constraint back into the Lagrangian by means of a Lagrange multiplier $\lambda$; one then gets

$\mathscr{L}=-\phi_{k}^{a} \dot{\Gamma}_{k}^{a}+\pi_{\mu}^{a} \dot{A}^{a \mu}+\dot{\lambda}^{a}\left(\pi_{0}^{a}+D_{k}^{a b} \phi_{k}^{b}\right)-\mathscr{V}^{(1)}$,

where the first-iterated potential density is $\mathscr{V}^{(1)}=\left.\mathscr{V}^{(0)}\right|_{\chi=0}$. From the above Lagrangian we may notice that the field $\Gamma_{0}$ naturally disappears when the constraint is taken as a strong relation. Now, in the first-iterate case, the symplectic variables are $\xi_{\alpha}^{(1)}=\left\{A_{k}^{a}, \pi_{k}^{a}, A_{0}^{a}, \pi_{0}^{a}, \Gamma_{k}^{a}, \phi_{k}^{a}, \lambda^{a}\right\}$, and we have the following one-form:

$$
\begin{gathered}
\Gamma a_{i}^{(1)}=-\phi_{i}^{a}, \quad{ }^{A} a_{i}^{(1)}=-\pi_{i}^{a}, \quad{ }^{A_{0}} a^{(1)}=\pi_{0}^{a}, \\
\lambda a^{(1)}=\pi_{0}^{a}+D_{k}^{a b} \phi_{k}^{b} .
\end{gathered}
$$

By evaluating the corresponding matrix elements, the symplectic two-form matrix reads

$$
\text { (1) } f(x, y)=\left[\begin{array}{cc}
\mathbf{A}_{i j}^{a b} & \mathbf{D}_{j}^{a b} \\
-\left(\mathbf{D}^{T}\right)_{i}^{b a} & \mathbf{C}_{i j}^{a b}
\end{array}\right] \delta(x, y) \text {, }
$$

with

$$
\begin{aligned}
\mathbf{C}_{i j}^{a b} & =\left[\begin{array}{ccc}
0 & \delta^{a b} \delta_{i j} & 0 \\
-\delta^{a b} \delta_{i j} & 0 & \left(D_{y}\right)_{i}^{b a} \\
0 & \left(D_{x}\right)_{i}^{a b} & 0
\end{array}\right], \\
\mathbf{D}_{i}^{a b} & =\left[\begin{array}{ccc}
0 & 0 & -g f^{a b c} \phi_{i}^{c} \\
0 & 0 & 0 \\
0 & 0 & 0 \\
0 & 0 & \delta^{a b}
\end{array}\right] .
\end{aligned}
$$

Again, we see that the first-iterated symplectic is singular. In the next step we should determine its eigenvector with zero eigenvalue. From that it follows that

$$
\bar{v}_{\alpha}=\left(\mathbf{0},\left(\bar{v}_{2}\right)_{k}^{a},\left(\bar{v}_{3}\right)^{a}, 0,\left(\bar{v}_{5}\right)_{k}^{a}, \mathbf{0},\left(\bar{v}_{7}\right)^{a}\right) .
$$

Therefore, from the eigenvector $\bar{v}_{\alpha}$ (64) we can evaluate the consistence condition and get

$$
\begin{gathered}
\int \mathrm{d} x \nu_{7}^{b}(x)\left[\left(D_{k}\right)^{b c}\left(\pi^{c k}-g f^{c d e} \phi^{d k} A_{0}^{e}\right)\right. \\
\left.+g f^{b d c} \phi_{k}^{d} W_{0 k}^{c}\right](x)=0
\end{gathered}
$$

and, since $\nu_{7}^{a}$ is an arbitrary function, we obtain the constraint

$\bar{\chi}^{b} \equiv\left(D_{k}\right)^{b c}\left(\pi^{c k}-g f^{c d e} \phi^{d k} A_{0}^{e}\right)+g f^{b d c} \phi_{k}^{d} W_{0 k}^{c}=0$,

which is nothing more than the non-Abelian version of the Gauss law. Proceeding, we should introduce it back to the Lagrangian as a strong relation; thus the second-iterated Lagrangian reads

$$
\begin{aligned}
\mathscr{L}= & \dot{\eta}^{b}\left(\left(D_{k}\right)^{b c}\left(\pi^{c k}-g f^{c d e} \phi^{d k} A_{0}^{e}\right)+g f^{b d c} \phi_{k}^{d} W_{0 k}^{c}\right) \\
& -\phi_{k} \dot{\Gamma}_{k}+\pi_{\mu} \dot{A}^{\mu}+\dot{\lambda}\left(\pi_{0}^{a}+\left(D_{k} \phi_{k}\right)^{a}\right)-\mathscr{V}^{(2)}
\end{aligned}
$$

whereas the second-iterated potential density is given by $\mathscr{V}^{(2)}=\left.\mathscr{V}^{(1)}\right|_{\bar{\chi}=0}$ with

$$
\begin{aligned}
& \mathscr{V}^{(2)}=-\pi_{k}^{a} \Gamma_{k}^{a}-\frac{M^{2}}{2} \phi_{k}^{a} \phi_{k}^{a}-\frac{1}{6 M^{2}}\left(D_{0} W^{n m}\right)^{a}\left(D^{0} W_{n m}\right)^{a} \\
& +\phi_{k}^{a}\left(\frac{1}{3}\left(D^{m} W_{m k}\right)^{a}+g f^{a b c} A_{0}^{b} \Gamma_{k}^{c}+g f^{a b c} A_{0}^{b} W_{0 k}^{c}\right) \\
& +\frac{1}{4} W_{k m}^{a} W^{a k m}-\frac{1}{2}\left(\Gamma_{k}^{a}-\partial_{k} A_{0}^{a}+g f^{a b c} A_{0}^{b} A_{k}^{c}\right)^{2} \\
& -\frac{1}{6 M^{2}}\left[\left(D_{r} W^{n m}\right)^{a}\left(D^{r} W_{m m}\right)^{a}+\left(D_{r} W^{r 0}\right)^{a}\left(D^{m} W_{m 0}\right)^{a}\right. \\
& \left.+2\left(D_{m} W^{0 n}\right)^{a}\left(D^{m} W_{0 n}\right)^{a}+\frac{2}{3}\left(D_{m} W^{m n}\right)^{a}\left(D^{r} W_{r n}\right)^{a}\right] \\
& +\frac{g}{18 M^{2}} f^{a b c}\left[3 W_{0 m}^{a} W_{m k}^{b} W_{k 0}^{c}-W_{k m}^{a} W_{m j}^{b} W_{j k}^{c}\right] .
\end{aligned}
$$

From the above second-iterated Lagrangian we read off the following vectors:

$$
\begin{aligned}
\Gamma a_{i}^{(2)}= & -\phi_{i}, \quad{ }^{A} a_{i}^{(2)}=-\pi_{i}, \quad{ }^{A_{0}} a^{(2)}=\pi_{0}, \\
& \lambda a^{(2)}=\pi_{0}^{a}+\left(D_{k} \phi_{k}\right)^{a}, \\
\eta a^{(2)}= & \left(D_{k}\right)^{b c}\left(\pi^{c k}-g f^{c d e} \phi^{d k} A_{0}^{e}\right)+g f^{b d c} \phi_{k}^{d} W_{0 k}^{c} ;
\end{aligned}
$$

these results lead to the corresponding two-form matrix for $\xi_{\alpha}^{(2)}=\left\{A_{k}^{a}, \pi_{k}^{a}, A_{0}^{a}, \pi_{0}^{a}, \Gamma_{k}^{a}, \phi_{k}^{a}, \lambda, \eta\right\}:$

$$
{ }^{(2)} f(x, y)=\left[\begin{array}{cc}
\mathbf{A}_{i j}^{a b} & \mathbf{E}_{j, x}^{a b} \\
-\left(\mathbf{E}^{T}\right)_{i, y}^{b a} & \mathbf{F}_{i j}^{a b}
\end{array}\right] \delta(x, y),
$$


with

$$
\begin{aligned}
& \mathbf{E}_{i, x}^{a b}=\left[\begin{array}{cccc}
0 & 0 & -g f^{a b c} \phi_{i}^{c} & (2)(A, \eta) \\
0 & 0 & 0 & f^{a b} \\
0 & 0 & 0 & \left(D_{x}^{i}\right)^{b a} \\
0 & 0 & \delta^{a b} & 0
\end{array}\right],
\end{aligned}
$$

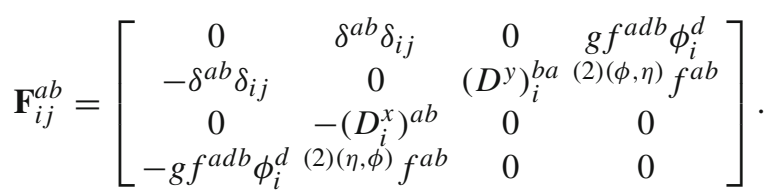

We have again obtained a singular matrix, now the seconditerated one ${ }^{(2)} f(x, y)$. Next, we determine the zero-mode vectors, which now consist in a set of two vectors,

$\tilde{v}_{\alpha}=\left(\left(\overline{\bar{v}}_{1}\right)_{k}^{a}, \mathbf{0}, 0,\left(\overline{\bar{v}}_{4}\right)^{a}, \mathbf{0},\left(\overline{\bar{v}}_{6}\right)_{k}^{a}, 0,\left(\overline{\bar{v}}_{8}\right)^{a}\right)$

and

$\overline{\bar{v}}_{\alpha}=\left(\mathbf{0},\left(\overline{\bar{v}}_{2}\right)_{k}^{a},\left(\overline{\bar{v}}_{3}\right)^{a}, 0,\left(\overline{\bar{v}}_{5}\right)_{k}^{a}, \mathbf{0},\left(\overline{\bar{v}}_{7}\right)^{a}, 0\right)$.

However, the vector $\overline{\bar{v}}_{\alpha}$ generates the constraint $\bar{\Omega}^{b}(x) \equiv$ $\left(D_{k}\right)^{b c}\left(\pi^{c k}-g f^{c d e} \phi^{d k} A_{0}^{e}\right)+g f^{b d c} \phi_{k}^{d} W_{0 k}^{c}=0$. Therefore, the vector $\tilde{v}_{\alpha}$ only is of our interest; but this zero mode does not generate any new constraints and, consequently, the symplectic matrix remains singular. Therefore, there are gauge degrees of freedom to be fixed. A suitable choice here is the generalized Coulomb gauge $\left(1+M^{-2} \square\right) \partial^{k} A_{k}^{a}=0, \Gamma_{0}^{a}=0$ and $A_{0}^{a}=0$. The same set of constraints obtained here was previously found in [43] through an analysis à la Dirac, where the generator of the gauge symmetry was also discussed. This shows again the agreement between the Dirac and FaddeevJackiw methods.

\subsection{Transition amplitude via BFV}

Instead of evaluating the inverse of the third-iterated twoform matrix, and then determine the generalized brackets between the dynamical fields, and proceed to the quantization by the correspondence principle, we shall rather work in a path-integral framework. Therefore, we shall now compute the transition amplitude through the BFV formalism [4042], because, though there is a proposal relating the pathintegral to the FJ method [38,39], it is not clear that the method works and is consistent with a gauge theory. We see that the transition amplitude in our case is written

$$
\begin{aligned}
\mathscr{Z}= & \int D A_{k}^{a} D \pi_{k}^{a} D \Gamma_{m}^{b} D \phi_{m}^{b} D \lambda^{c} D b^{c} D \bar{c}^{d} D c^{d} D \bar{P}^{e} D P^{e} \\
& \times \exp \left[i \int \mathrm { d } ^ { 4 } x \left\{\pi_{k}^{a} \dot{A}^{a k}+\phi_{k}^{a} \dot{\Gamma}^{a k}+\dot{c}^{a} \bar{P}^{a}+\left(\partial_{0} \bar{c}\right)^{d} P^{d}\right.\right. \\
& \left.\left.+\dot{\lambda}^{a} b^{a}-\mathscr{V}^{(3)}\right\}+i \int \mathrm{d} x_{0}\left\{\Psi, Q_{B R S T}\right\}\right]
\end{aligned}
$$

where $\mathscr{V}^{(3)}$ is recognized as being the canonical Hamiltonian in the first-order approach, and it is given by (68); we have

$\mathscr{V}^{(3)}=\left.\mathscr{V}^{(2)}\right|_{\Omega=0}$.

$\Omega$ consists in the generalized Coulomb gauge

$$
\left(1+M^{-2} \square\right) \partial^{k} A_{k}^{a}=0, \quad \Gamma_{0}^{a}=0, \quad A_{0}^{a}=0 .
$$

Furthermore, $\left(c_{a}, \bar{P}_{a}\right)$ and $\left(\bar{c}_{a}, P_{a}\right)$, are the pairs of ghost fields and their respective momenta, while $\left(\lambda_{a}, b_{a}\right)$ is a Lagrange multiplier and its momentum, all satisfying the following Berezin brackets:

$\left\{\bar{c}_{a}(z), P_{b}(w)\right\}_{B}=\delta_{a b} \delta(z, w)$,

$\left\{\bar{P}_{a}(z), c_{b}(w)\right\}_{B}=-\delta_{a b} \delta(z, w)$,

$\left\{\lambda_{a}(z), b_{b}(w)\right\}_{B}=\delta_{a b} \delta(z, w)$.

In the expression (75) it remains to define two quantities. The first is the BRST charge, which with the full set of constraints is written

$$
\begin{aligned}
Q_{\mathrm{BRST}}= & \int \mathrm{d}^{3} x\left[c^{b}\left(\left(D_{k} \pi^{k}\right)^{b}+g f^{b d c} \phi_{k}^{d} \Gamma_{k}^{c}\right)\right. \\
& \left.-i P^{a} b^{a}+\frac{1}{2} \bar{P}^{a} f^{a b c} c^{b} c^{c}\right],
\end{aligned}
$$

whereas we see that the gauge-fixing function $\Psi$, in the generalized Coulomb gauge, reads

$$
\begin{aligned}
\Psi= & \int \mathrm{d}^{3} z\left[\frac{i \xi}{2} b^{a} \bar{c}^{a}+i \bar{c}^{a}\left(1+M^{-2} \square\right) \partial^{k} A_{k}^{a}\right. \\
& \left.-\lambda^{a}\left(1+M^{-2} \square\right)^{-1} \bar{P}^{a}\right] .
\end{aligned}
$$

From the above expressions, it is not difficult to evaluate

$$
\begin{aligned}
& \left\{\Psi, Q_{\mathrm{BRST}}\right\} \\
& =\int \mathrm{d}^{3} z\left\{\frac{\xi}{2} b^{a} b^{a}+i \partial^{k} \bar{c}^{e}\left(1+M^{-2} \square\right)\left(D_{k} c\right)^{e}\right. \\
& \quad+b^{a}\left(1+M^{-2} \square\right) \partial^{k} A_{k}^{a}+i\left(1+M^{-2} \square\right)^{-1} \bar{P}^{a} P^{a} \\
& \quad+f^{a b c}\left(1+M^{-2} \square\right)^{-1} \bar{P}^{a} \lambda^{b} c^{c} \\
& \left.\quad+\left(1+M^{-2} \square\right)^{-1} \lambda^{a}\left(\left(D_{k} \pi^{k}\right)^{b}+g f^{b d c} \phi_{k}^{d} \Gamma_{k}^{c}\right)\right\} .
\end{aligned}
$$

Thus, substituting the result (81) into the transition-amplitude expression (75), and performing the variables integration and after some algebraic manipulation, one finds 


$$
\begin{aligned}
\mathscr{Z}= & \int D A_{\mu}^{a} D \bar{c}^{d} D c^{d} \\
& \times \exp \left[i \int \mathrm { d } ^ { 4 } z \left\{\mathscr{L}_{\mathrm{AAB}}+i \partial_{\mu} \bar{c}^{e}\left(1+M^{-2} \square\right)\left(D^{\mu} c\right)^{e}\right.\right. \\
& \left.\left.-\frac{1}{2 \xi}\left[\left(1+M^{-2} \square\right) \partial^{\mu} A_{\mu}^{a}\right]^{2}\right\}\right]
\end{aligned}
$$

Hence, from the BFV formalism we have obtained directly the desirable covariant expression for the transition amplitude. Furthermore, we see that the ghosts fields are coupled from the gauge fields, and matter fields may also be included.

\section{Concluding remarks}

In this paper we have presented a canonical study of higher-derivative theories, the Podolsky electrodynamics and its non-Abelian extension, the Alekseev-Arbuzov-Baikov effective Lagrangian, in the point of view of the symplectic Faddeev-Jackiw approach. Although the Dirac method remains as the standard method to deal with constrained systems, it has been recognized that some calculation is unnecessarily cumbersome there, and then it is exactly in this point where the FJ method shows to be an economical and rich framework for first-order Lagrangian functions, obviating mainly unnecessary calculations.

At the beginning we have reviewed briefly the main aspects of the symplectic FJ method. Subsequently, we applied the method to studying the generalized electrodynamics. The full set of the known constraints [30] was obtained, and afterwards it was showed that the third zeromode vector does not generate any new constraint, however, the symplectic matrix remained singular, an imprint characteristic of gauge theories. Therefore, the gauge had to be fixed; to attain that we have chosen to work with the generalized Coulomb gauge. From all that we were able to obtain a non-singular symplectic matrix, and by evaluating the inverse of such a matrix, we obtained the generalized brackets between the dynamical fields, in accordance with the previous results of Dirac's approach in [30]. Moreover, next we introduced the AAB's effective Lagrangian. The lines in studying this non-Abelian theory followed those presented to the generalized electrodynamics. Again, the known full set of constraints was obtained to be in accordance with the result from the Dirac approach [43]. As happens in gauge theories, the third zero-mode vector does not generate any new constraint and the symplectic matrix remained singular. However, instead of using the usual prescription and introduce new constraints, in order to fix the gauge degrees of freedom, we had chosen to quantize the theory via path-integral methods. Although there is a proposal of the FJ method in the path-integral framework, its content it is not clear for gauge theories. Therefore, we followed the well-known BFV method to construct the transition amplitude.

It was successfully showed here that the symplectic approach of Faddeev-Jackiw works perfectly well also for higher-derivative theories, and that all the obtained results were in accordance with previous ones when the Dirac methodology was applied. This is somehow in contrast with the assertion [15] that the FJ method produces constraints that do not exist in the Dirac theory. Furthermore, this emphasizes that in fact the FJ method can be considered as a good candidate of a framework where a deeper analysis may be performed, especially in more intriguing theories, such as General Relativity and renormalizable higher-derivative proposals of a quantum theory of Gravity, in different dimensionality, where the constraint analysis is not always easy to accomplish and is not always clear within the Dirac methodology. These issues and others will be further elaborated, investigated, and reported elsewhere.

Acknowledgments RB thanks FAPESP for full support, BMP thanks $\mathrm{CNPq}$ and CAPES for partial support.

Open Access This article is distributed under the terms of the Creative Commons Attribution License which permits any use, distribution, and reproduction in any medium, provided the original author(s) and the source are credited.

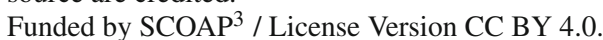

\section{References}

1. P. A. M. Dirac, Lectures on Quantum Mechanics (Yeshiva University, New York, 1964)

2. K. Sundermeyer, Constrained Dynamics. Lectures Notes in Physics, Vol. 169 (Springer-Verlag, New York, 1982)

3. L. Faddeev, R. Jackiw, Phys. Rev. Lett. 60, 1692 (1988)

4. J. Barcelos-Neto, C. Wotzasek, Mod. Phys. Lett. A 7, 1737 (1992)

5. J. Barcelos-Neto, C. Wotzasek, Int. J. Mod. Phys. A 7, 4981 (1992)

6. H. Montani, C. Wotzasek, Mod. Phys. Lett. A 8, 3387 (1993)

7. C. Wotzasek, Ann. Phys. 243, 76 (1995)

8. H.S. Blas, B.M. Pimentel, Ann. Phys. 282, 67 (2000)

9. E.M.C. Abreu, A.C.R. Mendes, C. Neves, W. Oliveira, R.C.N. Silva, C. Wotzasek, Phys. Lett. A 374, 3603 (2010)

10. E.M.C. Abreu, A.C.R. Mendes, C. Neves, W. Oliveira, R.C.N. Silva, JHEP 1306, 093 (2013)

11. M.E.V. Costa, H.O. Girotti, Phys. Rev. Lett. 60, 1771 (1988)

12. J. Govaerts, Int. J. Mod. Phys. A 5, 3625 (1990)

13. D.S. Kulshreshtha, H.J.W. Müller-Kirsten, Phys. Rev. D 43, 3376 (1991)

14. J. Antonio García, J.M. Pons, Int. J. Mod. Phys. A 12, 451 (1997)

15. L. Liao, Y.C. Huang, Ann. Phys. 322, 2469 (2007)

16. Y.C. Huang, J.L. Yanga, Phys. Lett. B 668, 438 (2008)

17. Y.C. Huang, L.-X. Yi, Ann. Phys. 325, 2140 (2010)

18. M. Ostrogradski, Mem. Ac. St. Petersburg VI 4, 385 (1850)

19. R. Weiss, Proc. R. Soc. A 169, 102 (1938)

20. J.S. Chang, Proc. Camb. Philos. Soc. 44, 76 (1948)

21. K. Stelle, Phys. Rev. D 16, 953 (1977)

22. K. Stelle, Gen. Relativ. Gravit. 9, 353 (1978)

23. E.S. Fradkin, A.A. Tseytlin, Nucl. Phys. B 201, 469 (1982)

24. P. Hořava, Phys. Rev. D 79, 084008 (2009) 
25. A. Pais, G.E. Uhlenbeck, Phys. Rev. 79, 145 (1950)

26. W. Heisenberg, Nucl. Phys. 4, 532 (1957)

27. B. Podolsky, Phys. Rev. 62, 68 (1942)

28. B. Podolsky, C. Kikuchy, Phys. Rev. 65, 228 (1944)

29. B. Podolsky, P. Schwed, Rev. Mod. Phys. 20, 40 (1948)

30. C.A.P. Galvão, B.M. Pimentel, Can. J. Phys. 66, 460 (1988)

31. R.R. Cuzinatto, C.A.M. de Melo, P.J. Pompeia, Ann. Phys. 322, 1211 (2007)

32. A.I. Alekseev, B.A. Arbuzov, Theor. Math. Phys. 59, 372 (1984)

33. M. Baker, J.S. Ball, F. Zachariasen, Nucl. Phys. B 229, 445 (1983)

34. M. Baker, L. Carson, J.S. Ball, F. Zachariasen, Nucl. Phys. B 229, 456 (1983)
35. C.-N. Yang, R.L. Mills, Phys. Rev. 96, 191 (1954)

36. R. Utiyama, Phys. Rev. 101, 1597 (1956)

37. A.I. Alekseev, B.A. Arbuzov, V.A. Baikov, Theor. Math. Phys. 52, 739 (1982)

38. L. Liao, Y.C. Huang, Phys. Rev. D 75, 025025 (2007)

39. L. Liao, Y.C. Huang, Eur. Phys. J. C 60, 481 (2009)

40. E.S. Fradkin, G.A. Vilkovisky, CERN-TH-2332 (1977)

41. I.A. Batalin, G.A. Vilkovisky, Phys. Lett. B 69, 309 (1977)

42. E.S. Fradkin, T.E. Fradkina, Phys. Lett. B 72, 343 (1978)

43. C.A.P. Galvão, J.B.T. Boechat, J. Math. Phys. 31, 448 (1990) 